\title{
Mismatch repair, molecular switches, and signal transoluction
}

\author{
Richard Fishel ${ }^{1}$ \\ Genetics and Molecular Biology Program, Department of Microbiology and Immunology, Kimmel Cancer Center, Thomas \\ Jefferson University, Philadel phia, Pennsylvania 19107 USA
}

The foundation of molecular switches in biology is grounded in translation elongation and cellular signal transduction. In these systems, guanine nucleotidebound proteins (G proteins) produce the ON and OFF signaling states that act as gates for downstream biochemical processes. Recent studies on the human mismatch repair reaction have suggested a si milar molecular switch that relies on adenine nucleotide-bound forms (A proteins) to produce an ON and OFF signaling state. In the field of signal transduction, the concept of a molecular switch is elementary whereas the biochemical processes of DNA repair appear foreign. Similarly, the field of DNA repair recognizes the complex machinery required for DNA manipulation events but regards biochemical signaling processes as essential cellular input but outside the genome juggernaut. The concept of a molecular switch as an integral step in mismatch repair should accelerate communication between these two fields toward a resolved and unified mechanism for biological signaling processes.

\section{Genetics of mismatch repair}

There are at least three ways in which mismatched nucleotides arise in DN A: (1) physical or chemical damage to the DNA and its precursors, such as deamination of 5-methyl-cytosine (Friedberg 1990); (2) misincorporation of nucleotides during DNA replication can yield mismatched base pairs as well as the insertion and deletion of nucleotides (for review, see Kolodner 1996; Modrich 1989, 1997); and (3) genetic recombination produces regions of heteroduplex DNA that may contain mismatched nucleotides when such heteroduplexes result from the pairing of two different parental DNA sequences (Holliday 1964). Mismatched nucleotides produced by each of these mechanisms are known to be repaired by enzyme systems that are both specific and overlapping (Friedberg 1990).

The most extensively studied system for mismatch repair (MMR) is the DNA adenine methylation (Dam)instructed pathway of Escherichia coli (for review, see Modrich 1989; Modrich and Lahue 1996). The Dam-in-

'E-MAIL rfishel@hendrix.jci.tju.edu; FAX (215) 923-1098. structed pathway promotes a long-patch $(\sim 2 \mathrm{~kb})$ excision repair reaction that is dependent genetically on the mutH, mutL, mutS, and mutU (uvrD) gene products. Discrimination of the newly replicated DNA strand from the original template DN A strand is dependent on transient undermethylation of the adenine nucleotide within a GATC Dam sequence. The MutHLS pathway appears to be the most active MMR pathway in E. coli and is known to both increase the fidelity of DNA replication as well as to act on recombination intermediates containing mispaired bases (Fishel and Kolodner 1983; Fishel et al. 1986).

Homologs of the prokaryotic M utS and M utL proteins have been identified in nearly every organism with the exception of Archaea (for review, see Kolodner 1996; Fishel and Wilson 1997). At present there are 41 MutS homol ogs and 21 MutL homologs in the NCBI database. In Saccharomyces cerevisiae, six MutS homologs (MSH 1-MSH6) and three MutL homologs (MLH1, MLH2, PM S1) have been identified, whereas in human cells a nearly identical set of five MutS homologs (hMSH2-hMSH6) and three MutL homologs (hM LH1, hPM S1, and hPM S2) are known (Fujii and Shimada 1989; Kramer et al. 1989; Linton et al. 1989; Mankovich et al. 1989; Reenan and Kolodner 1992; Fishel et al. 1993; N ew et al. 1993; Bronner et al. 1994; Burns et al. 1994; N icolaides et al. 1994; Prolla et al. 1994; Hollingsworth et al. 1995; Palombo et al. 1995; Acharya et al. 1996). Yet, outsi de of Gram-negative bacteria, there do not appear to be homologs of MutH. Thus, the mechanism of strand discrimination in even close relatives of $E$. coli, the gram-positive bacteria, remains a mystery. The multiple M utS and M utL homologs have been found to participate in the diverse activities of nuclear (MSH2, MSH3, MSH6, MLH1, PMS1) and organellar (M SH1) postreplication mismatch repair as well as distinct mei otic functions (M SH 4, M SH5) (Kolodner 1996; Fishel and Wilson 1997).

\section{Biochemistry of mismatch repair}

Purification and reconstitution studies by Modrich and colleagues have led to a biochemical model for postreplication mismatch repair in E. coli. The reconstituted 
system requires the $M$ utH, MutL, MutS, and UvrD (helicase II) proteins along with DNA polymerase III holoenzyme, DN A ligase, single-stranded DN A binding protein (SSB), and one of the single-stranded DN A exonucleases, Exo I, Exo VII, or Rec) (Lu et al. 1983; Su and Modrich 1986; Welsh et al. 1987; Grilley et al. 1989; Lahue et al . 1989; Cooper et al. 1993). In this widely held biochemical model, initiation of a MMR event occurs when MutS recognizes and binds mispaired nucleotides that result from polymerase misincorporation errors (Su and Modrich 1986). It is then suggested that MutS mismatch binding is foll owed by interaction with the M utL protein (Grilley et al. 1989), which has been proposed to accelerate an ATP-dependent translocation of the M utSMutL complex (Allen et al. 1997) to a hemimethylated GATC Dam site bound by M utH (Welsh et al. 1987). The MutS-M utL complex then stimulates an intrinsic endonuclease activity of $\mathrm{MutH}$, which results in a specific strand scission on the unmethylated, newly replicated DN A strand (Lahue et al. 1987; Welsh et al. 1987; Cooper et al. 1993). This strand scission directs one of three single-stranded exonucleases (Rec J, Exo I, Exo VII) to degrade the newly replicated strand, which is then resynthesized by the Pol III hol oenzyme complex (Lahue et al. 1989). The net result is a strand-specific mismatch repair event that can be bidirectional. Many of the genetic studies performed with this system appear to support this biochemical interpretation. For example, mutH, mutL, and mutS bacteria exhibit a mutator phenotype that is presumed to be the result of the increased probability of misincorporation errors leading to mutations (Demerec et al . 1957; Miyake 1960; Siegel and Bryson 1967; Hill 1970). However, not all predictions arising from this model agree with the genetic results. For example, rec exol exoVII bacteria do not appear to exhibit a mutator phenotype (Harris et al . 1998), suggesting that there may be other exonuclease(s) or mechanism(s) involved in the mismatch repair process.

\section{Unique functions for the mismatch repair proteins}

The most obvious unique function for mismatch repair proteins is the specific mispair binding activity ascribed to MutS homologs (Su and Modrich 1986; Fishel et al. 1994; Chi and Kolodner 1994; Drummond et al. 1995;
Acharya et al. 1996; Marsischky et al . 1996; Gradia et al. 1997). A clear function of the M utL homologs has not yet been identified. Classification of MutS and MutL homologs is based on the recognition of highly conserved regions of amino acid identity. The most highly conserved region of the $\mathrm{M}$ utS homologs is confined to a region of 150 amino acids that encompass a helix-turnhelix $(\mathrm{HTH})$ domain associated with a Walker-A adenine nucleotide and magnesium binding motif (Walker et al. 1982). This adenine nucleotide binding domain constitutes $100 \%$ of the identity between the known MutS homologs (Fishel and Wilson 1997; Fig. 1). Purified bacterial, yeast, and human MutS homologs have been found to possess an intrinsic low-level ATP hydrolysis (ATPase) activity (Haber and Walker 1991; Chi and Kolodner 1994; Alani et al . 1997; Gradia et al . 1997). This AT Pase activity is likely to be important for the function of the MutS homologs because mutation of a conserved lysine residue in the adenine nucl eotide binding domain results in a dominant mutator phenotype in both bacteria and yeast (Haber and Walker 1991; Alani et al. 1997). As suggested above, the most widely held model for MMR suggests that M utS mispair binding is foll owed by MutL association, which then results in an energy-dependent translocation of this complex to a hemimethylated Dam site that is occupied by the MutH protein. In retrospect, this appears to have been a simplistic view because the rate of ATP hydrolysis $\left(k_{\text {cat }} \cong 10 \mathrm{~min}^{-1}\right)$ is unlikely to be sufficient to drive mechanical translocation the several hundred to thousand nucleotides (on average) required to encounter a $\mathrm{MutH}$ bound hemimethylated site. For example, if one ATP was required to translocate one nucleotide (as the most well-accepted mechanism would suggest), then it would take 25-100 min to encounter a M utH on average. Yet, remethylation of the transiently hemimethylated Dam sites has been found to occur within 0.1-3 min of the passing replication fork (Campbell and Kleckner 1990). Although the ATPase activity could in theory be significantly faster in vivo, no stimulatory factor has been identified to date despite an extensive search. In addition, the prevailing mechanism does not adequately account for M utL function nor the highly conserved domains recognized between MutL homologs from bacteria to man (regions containing 100\% identity in 21 homologs).

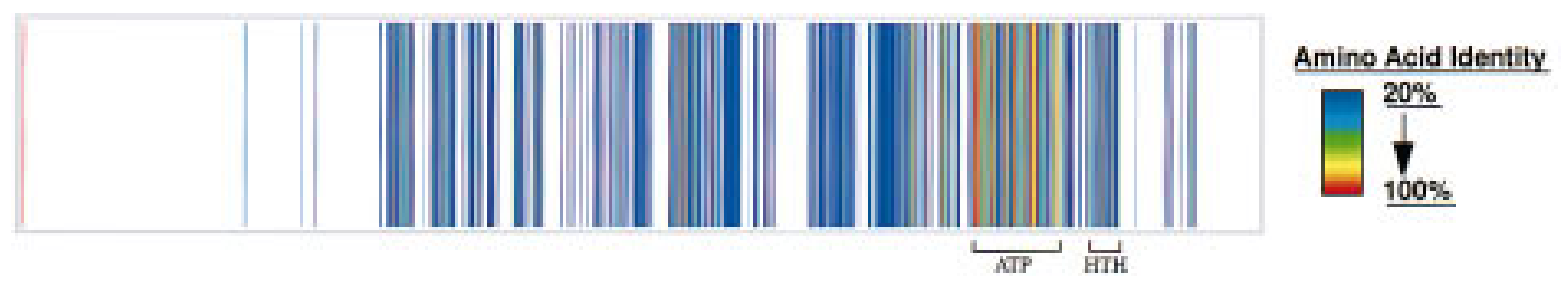

Figure 1. Homology spectrum of $35 \mathrm{M}$ utS homologs. Protein alignment was performed using Megalign (DNASTAR, Madison, WI). Alignments were colorized (DN ASTAR, Madison, WI) to indicate $20 \%$ (7/35 amino acid identity at an aligned amino acid position), up to $100 \%$ (35/35 amino acid identity). The consensus ATP binding domain (ATP) and HTH are shown. The initial alignment included 11 procaryotes and 9 eukaryotes. Whereas the prokaryotes generally contribute one MutS homolog, eukaryotes may contribute up to six different MutS homologs (Fishel and Wilson 1997). 


\section{The hMSH2-hMSH6 molecular switch}

Last year we proposed that the human MutS homologs, hM SH2-hM SH6, function as a mol ecular switch responsible for the timing of mismatch repair (Gradia et al. 1997; Fig. 2A,B). This hypothesis was based on the observations that: (1) The ADP-bound form has a high affinity for mismatched nucleotides; (2) the exchange of ADP for ATP results in the release from the mi smatch in the absence of hydrolysis; (3) rel ease appears to occur by simple dissociation and/or hydrolysis-independent diffusion off the ends of the short oligonucleotides used in these experiments; and (4) hydrolysis of ATP results in recovery of the mismatch-binding competent ADPbound form. The ratelimiting step and the ulti mate control of the hM SH2-hM SH 6 molecular switch is likely to be the ADP $\rightarrow$ ATP exchange, which is exceedingly inefficient in the absence of a mi smatched nucleotide. The characteristics of the hMSH2-hMSH 6 switch appear most similar to the G-protein mediators of seven-transmembrane (7-TM) domain receptor signaling such as that used by the $\beta$-adrenergic and rhodopsin receptors (Fig. 2C), as well as the prototypical oncoprotein/G-protein Ras (Fig. 2D; Tocque et al. 1997). M ore specifically, the observation that hMSH2-hMSH 6 is induced to exchange ADP for ATP in the presence of a mismatched nucleotide and then dissociates from the mismatch to transduce a signal, mirrors ligand binding by 7-TM receptors, which induce the associated $G$ protein to exchange GDP for GTP and then the GTP-bound form dissociates from the receptor to transduce a signal.

These similarities allowed us to develop two related models for mismatch repair that are fundamental ly different from all previously suggested mechanisms. These models are both based on the concept that MutS and its homologs are a novel type of molecular switch that determines the timing and/or appropriate assembly of repair components. The apparent affinity of hMSH2-
hMSH 6 for mismatched nucleotides $\left(K_{\mathrm{d}} \cong 2-20 \mathrm{~nm}\right)$ has suggested that a single mismatch in a human cell should be recognized efficiently and bound. Furthermore, this binding is stabilized slightly in the presence of ADP. We propose two nonexclusive models: In the first model, tight binding to mismatch nucleotides by the ADPbound form of hM SH2-hM SH 6 acts as a flag for the assembly or nearby localization of the excision repair machinery. When the complete system is assembled, then exchange of ADP for ATP would be triggered and hMSH2-hMSH 6 would be released from the mismatch (Fig. 2A); thus signaling exonucleolytic excision and resynthesis of the region containing the mismatched nucleotide. Once released from the mismatched nucleotides, the intrinsic ATPase activity of hM SH2-hMSH 6 would hydrolyze the bound ATP, resulting in a form that is once again competent for mismatch binding (Fig. 2A). In the second model, recognition of a mismatched nucleotide provokes the ADP $\rightarrow$ ATP nucleotide exchange and hydrolysi s-independent, DN A-associated diffusion of hMSH2-hMSH 6 away from the mismatch to the assembled (or partially assembled) mismatch repair components (Fig. 2B). Activation of these components by the confederation of this ATP-bound form of hMSH2hM SH 6 either engages the repair process (signaling the timing of mismatch repair as above) or all ows the appropriate assembly of the remaining mismatch repair machinery. This activation event would result in the release of hMSH2-hMSH6, hydrolysis of ATP, and recycling of the mismatch competent form (Fig. 2B). The advantage of this latter model is that the hM SH 2-hM S6 remains associated with the DNA in an activated-form poised to transduce the mismatch signal to any nearby mismatch repair components.

As a free protein complex, hM SH2-hMSH 6 does not efficiently exchange the ADP remaining after hydrolysis, providing a long-term mismatch-recognition-competent molecule. Again, this ADP-mismatch-bound and ATP-
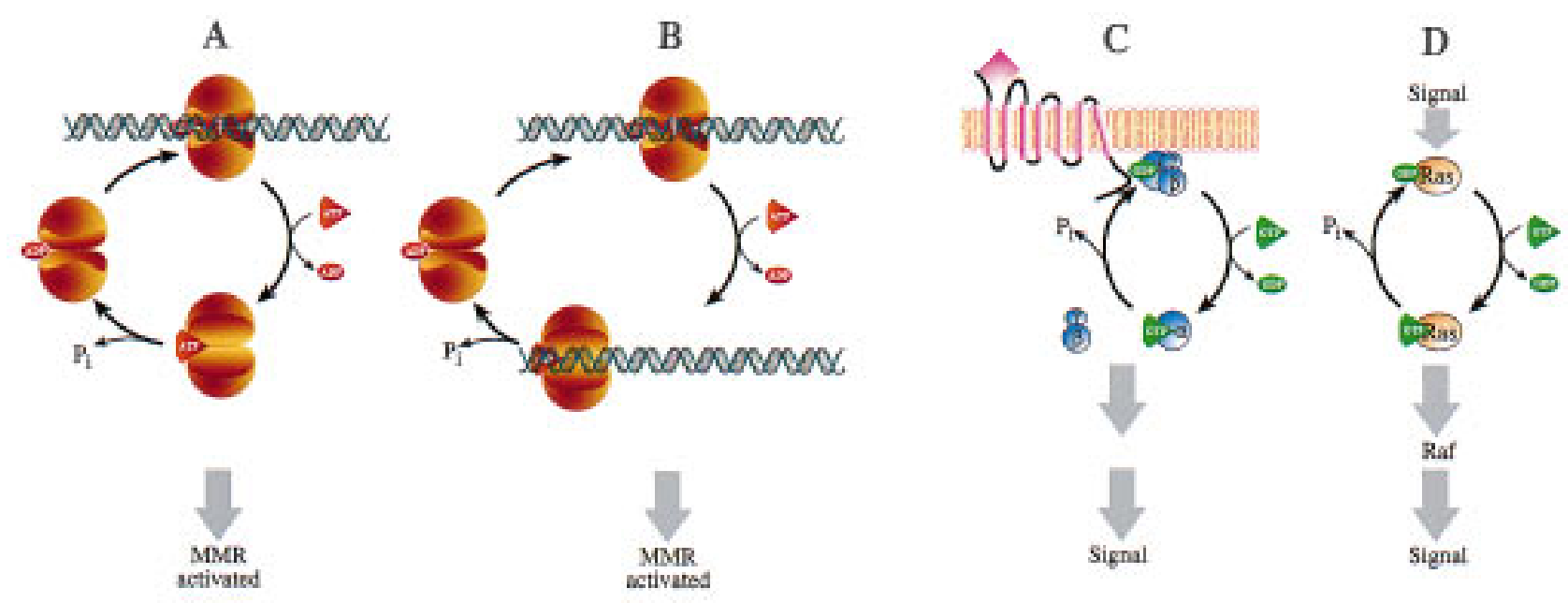

Figure 2. Comparison of the hM SH2-hM SH 6 molecular switch with G-protein switches. (A) The hM SH 2-hM SH 6 simple-dissociation molecular switch; (B) the hM SH2-hM SH 6 hydrolysis-independent translocation molecular switch; (C) the heterotrimeric Gprotein molecular switch; (D) the Ras G-protein molecular switch. 
mismatch-released switch appears functionally equivalent to the 7-TM G-protein switches in that a signal is transduced to downstream factors when the NDP is exchanged for the NTP and the G protein dissociates from the 7-TM receptor. The key difference in the mismatch repair models described above and those previously proposed, is the concept that ATP hydrolysis is not required to physically transduce the mismatch binding signal to the downstream machinery, but instead is required to recycle the recognition system. Furthermore, in these models the authentic signal is the conformational transition that ATP binding induces in the MutS homologs hMSH2-hMSH6; thus, the concept of a molecular switch.

\section{Switches in signal transduction}

G proteins are the prototypical biological switches (for review, see Sprang 1997 and references therein). Historically, the first recognized G protein was the E. coli EF-Tu subunit, which promotes the correct positioning of an aminoacyl-tRNA on an mRNA within the ribosome (Keller and Zamecnik 1955; N athans et al. 1962). As the process of protein synthesis proceeds, a more classic switch reaction occurs when a second G protein, EF-G, binds to GTP and signals the translocation of a growing peptidyl-tRN $A$ from the $A$ site to the $P$ site, prior to EF-Tu recruitment of the next aminoacyl tRNA. The hydrolysis of the bound GTP by EF-G is used to recycle/ reset the system. However, it was the Ras oncogene that focused attention on the function of $G$ proteins (Fig. 2D) (Weinberg 1989). In general, G proteins use the binding energy of GTP to stabilize "switch regions" in conformations that will permit their association with effectors, and the hydrolysis of GTP $\rightarrow$ GDP $+P_{i}$ is used to reset the switch. M utagenesis and $X$-ray crystal lography studies have identified five protein regions ( $G$ domains) that contact the GTP at points around the nucleoside rings and along the length of the phosphate chain (Sprang 1997). T wo of those $G$ domains include the Walker $A$ and B motifs (Walker et al. 1982). Furthermore, there are at least two switch regions (switch I and II) that undergo conformational transitions in response to nucleotide binding and hydrolysis have been identified in all of the G proteins with known crystal structure (Sprang 1997). Switch regions appear to be involved in making contact with effectors and/or regulators of the GTPase activity. These regions can be extremely malleable and have been shown to be involved in the physical association and dissociation of components in the signaling cascade (Sprang 1997).

One of the most important observations concerning $\mathrm{G}$ proteins is their regulation by associated proteins (Bokoch and Der 1993). There are two halves to the GT Pase cycle: $\gamma$-phosphate hydrolysis and GDP $\rightarrow$ GTP nucleotide exchange. Both of these steps can be regulated either by inhibition or acceleration of these partial reactions. For example, the Ras protein has an remarkably sluggish intrinsic GT Pase activity (T rahey et al. 1987), which can be accelerated at least $10^{4}$ - to $10^{5}$-fold by a GTPase acti- vating protein (GAP) (Trahey and McCormick 1987). In addition, there are other regulators of $G$ protein signaling (RGS) that singularly accel erate $\gamma$-phosphate hydrolysis, and GDP/GTP exchange factors (GEFs) and guanine dissociation inhibitors (GDIs) that singularly affect nucleotide exchange (Quilliam et al. 1995; Dohlman and Thorner 1997; Tocque et al. 1997). It is interesting to note that highly conserved amino acid domains have been identified within families of these $G$ protein regulators and their ubiquity suggests that similar factors may exist for the A proteins of which the hMSH2-hMSH6 mismatch repair switch would be a prototypical member. The most likely candidate for an adenine nucleotide regulator of the M utS homologs would be the M utL homologs, which would additionally account for the high conservation of M utL domains. Tests of this hypothesis are currently underway.

\section{Biological switches and the second law of thermodynamics}

One could argue that the concept of a singular ON or OFF state in a molecular switch might violate the Second Law of Thermodynamics. The Second Law requires that biochemical systems transit one state to the other by a series of microscopically reversible steps. This idea is based in statistical mechanics as it is applied to a system at equilibrium, which must be applied a priori to enzyme catalyzed biol ogical processes. It is easy to visualize the origins of the principle of microscopic reversibility by considering the consequences were it NOT true. For example, if the rate of $A \rightarrow B$ were greater than $B \rightarrow A$ at equilibrium, each of the rates $B \rightarrow C, C \rightarrow D$, and $D \rightarrow A$ would al so have to be greater than their re verse rates to prevent buildup of the concentration of any species, which is not permitted at equilibrium. In this case there would be a preferred direction-of-operation of the reaction cycle. Such a spontaneous cycle in a system at equilibrium (i.e., an engine that spontaneously produces work) is not consistent with the drive toward maximum entropy contained in the Second Law.

There is no violation of the Second Law if the transit from an OFF to ON state (or vice versa) occurs reversibly. The molecular basis for this type of microscopic reversibility can be visual ized for the hM SH2-hM SH 6 and Gprotein switches as reversible nucleotide binding as well as intermediate protein conformational changes that occur while transiting the extreme states. It is these conformational transitions that determine interaction with effectors which is ultimately pai $d$ for by the hydrolysis of NTP. M ore significantly, one can affect the equilibrium of each state experimentally by altering the ratio of NDP/NTP in the absence of any hydrolysis (see Fig. 6 in Gradia et al. 1997). It is also important to note that microscopic reversibility has been directly demonstrated for the gated maxi $\mathrm{K}^{+}$ion pump, a molecular switch controlled by similar conformational transitions (Song and Magleby 1994). Thus, molecular switches are both re versible and, at equilibrium, clearly preserving a fundamental tenant of thermodynamics. 


\section{The mamiage of signal transduction and DNA metabolism}

The use of controlled molecular switches appears to pervade all aspects of biology. From the standpoint of DNA metabolism, switch controlled processes appear mechanistically sensible. It is well known that the machines which perform replication, recombination, repair and chromosome segregation are very large and composed of multiple subunits (Alberts 1998). Just like the assembly line for an automobile or an airplane, the assembly of DN A metabolic machines must be done precisely and in a specific order to ensure appropriate function. A series of well defined switches could logically control the progression of such an ordered assembly process. Thus, the same type of switch-controlled cascade events that transduce cellular signals may also control DNA metabolic events. The only apparent difference between these switches is the nucleotide that induces the conformational transitions associated with signaling. At the moment the general rule seems to be guanine nucleotides for cellular signaling events and adenine nucleotides for DNA metabolic signaling events. This dogma raises a number of questions: Why purines? Why adenine versus guanine? Is there real ly generality to these observations? Do these switches signal more than just the assembly of DNA metabolic machines (i.e., are they signals of damage recognition, etc.)? Is there a connection between these DNA metabolic nucleotide-induced molecular switches and cell-cycle checkpoints? Clearly, communication between the fields of DNA repair and signal transduction will provide the breeding ground for appropriate experiments.

\section{Acknowledgments}

I thank Christoph Schmutte for the design and preparation of the figures; Lorena Kallal, Charles Brenner, and Philip Wedegaerdner for helpful discussions and criticism of the manuscript. This work was supported by $\mathrm{N}$ ational Institutes of Health grants CA56542 and CA67007.

\section{References}

Acharya, S., T. Wilson, S. Gradia, M .F. Kane, S. Guerrette, G.T. M arsischky, R. Kolodner, and R. Fishel. 1996. hM SH2 forms specific mispair-binding complexes with $\mathrm{hMSH} 3$ and hM SH6. Proc. Natl. Acad. Sci. 93,: 13629-13634.

Alani, E., T. Sokolsky, B. Studamire, J.J. Miret, and R.S. Lahue. 1997. Genetic and biochemical analysis of Msh2p-M sh6p: Role of ATP hydrolysis and M sh2p-M sh6p subunit interactions in mismatch base pair recognition. Mol. Cell. Biol. 17: 2436-2447.

Alberts, B. 1998. The cell as a collection of protein machinespreparing the next generation of molecular biologists. Cell 92: 291-294.

Allen, D.J., A. Makhov, M. Grilley, J. Taylor, R. Thresher, P. Modrich, and J.D. Griffith. 1997. MutS mediates heteroduplex loop formation by a transl ocation mechanism. EMBO J. 16: 4467-4476.

Bokoch, G.M. and C.J. Der. 1993. Emerging concepts in the Ras superfamily of GTP-binding proteins. FASEB J. 7: 750-759.
Bronner, C.E., S.M. Baker, P.T. Morrison, G. Warren, L.G. Smith, M.K. Lescoe, M. Kane, C. Earabino, J. Lipford, A. Lindblom et al. 1994. Mutation in the DN A mismatch repair gene homologue hM LH 1 is associated with hereditary nonpolyposis colon cancer. Nature 368: 258-261.

Burns, N., B. Grimwade, P.B. Ross-M acdonald, E. Choi, K. Finberg, G.S. Roeder, and M.S. Snyder. 1994. Large-scale analysis of gene expression, protein localization and gene disruption in Saccharomyces cerevisiae. Genes \& Dev. 8: 10871105.

Campbell, J.L. and N. Kleckner. 1990. E. coli oriC and the dnaA gene promoter are sequestered from dam methyltransferase following the passage of the chromosomal replication fork. Cell 62: 967-979.

Chi, N.W. and R.D. Kolodner. 1994. Purification and characterization of $\mathrm{MSH} 1$, a yeast mitochondrial protein that binds to DN A mismatches. J. Biol. Chem. 269: 29984-29992.

Cooper, D.L., R.S. Lahue, and P. Modrich. 1993. Methyl-directed mismatch repair is bidirectional. J. Biol. Chem. 268: 11823-11829.

Demerec, M., E.L. Lahr, T. Miyake, E. Galehran, E. Bal binder, S. Baric, K. Hashimoto, E.V. Glanville, and J.D. Gross. 1957. Bacterial genetics. Carnegie Inst. Wash. Yearbook 370: 390406.

Dohlman, H.G. and J. Thorner. 1997. RGS proteins and signaling by heterotrimeric G proteins. J. Biol. Chem. 272: 38713874.

Drummond, J.T., G.-M . Li, M.J. Longley, and P. M odrich. 1995. Isolation of an hM SH2-p160 heterodimer that restores DN A mismatch repair to tumor cells. Science 268: 1909-1912.

Fishel, R., A. Ewel, S. Lee, M.K. Lescoe, and J. Griffith. 1994. Binding of mismatched microsatellite DNA sequences by the human MSH2 protein. Science 266: 1403-1405.

Fishel, R., M.K. Lescoe, M.R. Rao, N.G. Copeland, N.A. Jenkins, J. Garber, M. Kane, and R. Kolodner. 1993. The human mutator gene homolog M SH 2 and its association with hereditary nonpolyposis colon cancer. Cell 75: 1027-1038.

Fishel, R. and T. Wilson. 1997. MutS homologs in mammalian cells. Curr. Opin. Genet. Dev. 7: 105-113.

Fishel, R.A. and R. Kolodner. 1983. Gene conversion in Escherichia coli: The identification of two repair pathways for mismatched nucleotides. U CLA Symp. Mol. Cell. Biol. New Ser. 11: 309-324.

Fishel, R.A., E.C. Siegel, and R. Kolodner. 1986. Gene conversion in Escherichia coli. Resolution of heteroallelic mismatched nucleoti des by co-repair. J. Mol. Biol. 188: 147-157.

Friedberg, E.C. 1990. DNA repair W.H. Freeman, New York, NY.

Fujii, H. and T. Shimada. 1989. Isolation and characterization of cDN A clones derived from the divergently transcribed gene in the region upstream from the human dihydrofolate reductase gene. J. Biol. Chem. 264: 10057-10064.

Gradia, S., S. Acharya, and R. Fishel. 1997. The human mismatch recognition complex hM SH2-hM SH 6 functions as a novel molecular switch. Cell 91: 995-1005.

Grilley, M., K.M. Welsh, S.S. Su, and P. M odrich. 1989. Isolation and characterization of the Escherichia coli mutL gene product. J. Biol. Chem. 264: 1000-1004.

Haber, L.T. and G.C. Walker. 1991. Altering the conserved nucleotide binding motif in the Salmonella typhimurium MutS mismatch repair protein affects both its ATPase and mismatch binding activities. EMBO J. 10: 2707-2715.

Harris, R.S., K.J. Ross, M.J. Lombardo, and S.M. Rosenberg. 1998. Mismatch repair in Escherichia coli cells lacking single-strand exonucleases Exol, ExoVII, and RecJ. J. Bacteriol. 180: 989-993. 
Hill, R.F. 1970. Location of genes controlling excision repair of UV damage and mutator activity in Escherichia coli WP2. Mutat. Res. 9: 341-344.

Holliday, R.A. 1964. A mechanism for gene conversion in fungi. Genet. Res. 5: 282-304.

Hollingsworth, N.M., L. Ponte, and C. Halsey. 1995. MSH5, a novel MutS homolog, facilitates meiotic reciprocal recombination between homologs in Saccharomyces cerevisiae but not mismatch repair. Genes \& Dev. 9: 1728-1739.

Keller, E.B. and P.C. Zamecnik. 1955. The effect of guanosine diphosphate and triphosphate on the incorporation of label ed amino acids into proteins. J. Biol. Chem. 221: 45-59.

Kolodner, R. 1996. Biochemistry and genetics of eukaryotic mismatch repair. Genes \& Dev. 10: 1433-1442.

Kramer, W., B. Kramer, M.S. Williamson, and S. Fogel. 1989. Cloning and nucleotide sequence of DNA mismatch repair gene PMS1 from Saccharomyces cerevisiae: Homology of PMS1 to procaryotic MutL and HexB. J. Bacteriol. 171: 5339-5346.

Lahue, R.S., K.G. Au, and P. Modrich. 1989. DNA mismatch correction in a defined system. Science 245: 160-164.

Linton, J.P., J.Y. Yen, E. Selby, Z. Chen, J.M. Chinsky, K. Liu, R.E. Kellems, and G.F. Crouse. 1989. Dual bidirectional promoters at the mouse dhfr locus: Cloning and characterization of two mRNA classes of the divergently transcribed Rep-1 gene. Mol. Cell. Biol. 9: 3058-3072.

Lu, A.L., S. Clark, and P. M odrich. 1983. M ethyl-directed repair of DN A base-pair mismatches in vitro. Proc. Natl. Acad. Sci. 80: 4639-4643.

Mankovich, J.A., C.A. Mclntyre, and G.C. Walker. 1989. $\mathrm{N}$ ucleotide sequence of the Salmonella typhimurium mutL gene required for mismatch repair: Homology of MutL to HexB of Streptococcus pneumoniae and to PMS1 of the yeast Saccharomyces cerevisiae. J. Bacteriol. 171: 5325-5331.

Marsischky, G.T., N. Filosi, M.F. Kane, and R. Kolodner. 1996. Redundancy of Saccharomyces cerevisiae M SH 3 and M SH 6 in MSH2-dependent mismatch repair. Genes \& Dev. 10: 407-420.

Miyake, T. 1960. Mutator factor in Salmonella typhimurium. Genetics 45: 755-762.

Modrich, P. 1989. M ethyl-directed DN A mismatch correction. J. Biol. Chem. 264: 6597-6600.

- - 1997. Strand-specific mismatch repair in mammalian cells. J. Biol. Chem. 272: 24727-24730.

Modrich, P. and R. Lahue. 1996. Mismatch repair in replication fidelity, genetic recombination, and cancer biology. Annu. Rev. Biochem. 65: 101-133.

Nathans, D., G. Von Ehrenstein, R. Monro, and F. Lipmann. 1962. Protein synthesis from aminoacyl-soluble ribonucleic acid. Fed. Proc. 21: 127-133.

$\mathrm{New}$, L., K. Liu, and G.F. Crouse. 1993. The yeast gene MSH3 defines a new class of eukaryotic MutS homologues. Mol. \& Gen. Genet. 239: 97-108.

Nicolaides, N.C., N. Papadopoulos, B. Liu, Y.F. Wei, K.C. Carter, S.M. Ruben, C.A. Rosen, W.A. Haseltine, R.D. Fleischmann, C.M. Fraser et al. 1994. Mutations of two PMS homologues in hereditary nonpolyposis colon cancer. Nature 371: 75-80.

Pal ombo, F., P. Gallinari, I. Iaccarino, T. Lettieri, M. Hughes, A. D'A rrigo, O. Truong, J.J. Hsuan, and J. Jiricny. 1995. GTBP, a 160-kilodalton protein essential for mismatch binding activity in human cells. Science 268: 1912-1914.

Prolla, T.A., D.-M. Christie, and R.M. Liskay. 1994. A requirement in yeast DNA mismatch repair for MLHI and PMS1, two homologs of the bacterial mutL gene. Mol. Cell. Biol. 14: $407-415$.
Quilliam, L.A., R. Khosravi Far, S.Y. Huff, and C.J. Der. 1995. Guanine nucleotide exchange factors: Activators of the Ras superfamily of proteins. BioEssays 17: 395-404.

Reenan, R.A.G. and R.D. Kolodner. 1992. Isolation and characterization of two Saccharomyces cerevisiae genes encoding homologs of the bacterial HexA and MutS mismatch repair proteins. Genetics 132: 963-973.

Siegel, E.C. and V. Bryson. 1967. Mutator gene of Escherichia coli Brit. J. Bacteriol. 94: 38-47.

Song, L. and K.L. M agl eby. 1994. Testing for microscopic reversibility in the gating of maxi $\mathrm{K}+$ channels using two-dimensional dwell-time distributions [published erratum appears in Biophys. J. 1994 Oct; 67(4):1792]. Biophy. J. 67: 91-104.

Sprang, S.R. 1997. G protein mechanisms: Insights from structural analysis. Annu. Rev. Biochem. 66: 639-678.

Su, S.-S. and P. Modrich. 1986. Escherichia coli mutS-encoded protein binds to mismatched DNA base pairs. Proc. Natl. Acad. Sci. 83: 5057-5061.

Tocque, B., I. Delumeau, F. Parker, F. Maurier, M.C. Multon, and F. Schweighoffer. 1997. Ras-GTPase activating protein (GAP): A putative effector for Ras. Cell Signal 9: 153-158.

Trahey, M. and F. McCormick. 1987. A cytoplasmic protein stimulates normal $\mathrm{N}$-ras p21 GTPase, but does not affect oncogenic mutants. Science 238: 542-545.

Trahey, M., R.J. Milley, G.E. Cole, M. Innis, H. Paterson, C.J. Marshall, A. Hall, and F. McCormick. 1987. Biochemical and biological properties of the human $\mathrm{N}$-ras p21 protein. Mol. Cell. Biol. 7: 541-544.

Walker, J.E., M. Saraste, M.J. Runswick, and N.G. Gay. 1982. Distantly related sequences in the al pha-and beta-subunits of ATP synthase, myosin, kinases and other ATP-requiring enzymes and a common nucleotide binding fold. EMBO J. 1: $945-951$.

Welsh, K.M., A.L. Lu, S. Clark, and P. Modrich. 1987. Isolation and characterization of the Escherichia coli mutH gene product. J. Biol. Chem. 262: 15624-15629.

Weinberg, R.A. 1989. Oncogenes, antioncogenes, and the molecular basis of multistep carcinogenesis. Cancer Res. 49: $3713-3721$. 


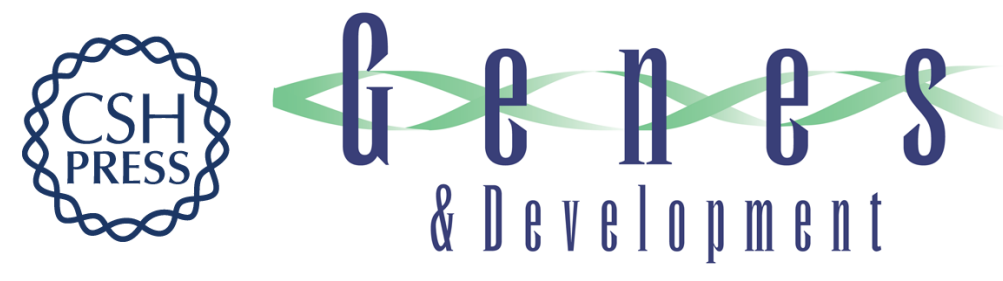

\section{Mismatch repair, molecular switches, and signal transduction}

Richard Fishel

Genes Dev. 1998, 12:

Access the most recent version at doi:10.1101/gad.12.14.2096

References This article cites 55 articles, 32 of which can be accessed free at: http://genesdev.cshlp.org/content/12/14/2096.full.html\#ref-list-1

License

Email Alerting Receive free email alerts when new articles cite this article - sign up in the box at the top Service right corner of the article or click here.

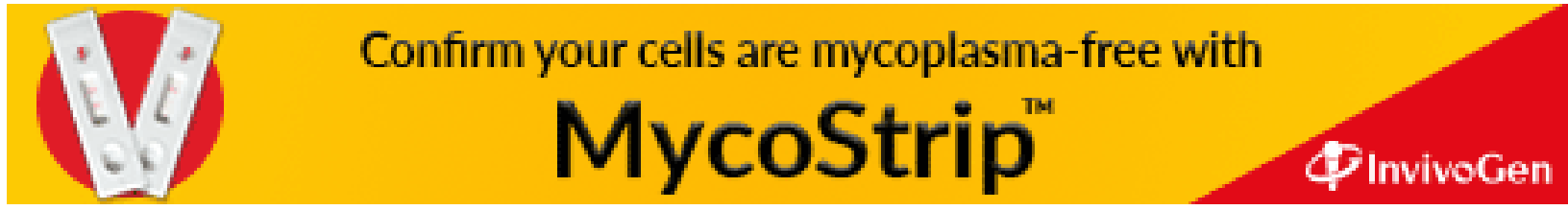

